\title{
Teaching Resource Organization and Scheduling Based on Grid Technology
}

\author{
Ding Wenhui \\ Guangdong University of Science and Technology
}

Keywords: Grid; Teaching; Dispatch; Organization

\begin{abstract}
The teaching resources in grid environment is distributed, heterogeneous and dynamic characteristics, for the massive teaching resources, resource organization and scheduling model is formulated via the characteristics of grid technology, the grid resources according to the specific rules of unified organization, scheduling to meet user needs is an urgent need to solve the problem. And a local--global teaching resource organization and scheduling model is proposed in view of the problems existing in process scheduling of grid resources, the model avoid blindness of resource query, improve the retrieval speed of resources; at the same time, through the process of resource scheduling in constant monitoring, the retrieval results according to specific rules is provided to the user, greatly improving the efficiency of resources utilization, which has certain application value.
\end{abstract}

\section{Introduction}

With the rapid development of network technology, resource sharing has become an important way for people to get resources. The University Digital Library, teaching platform containing large amounts of text, audio and video information, formed a huge repository is resources are the main approach to all the teachers and students and scholars to acquire[1][2]. But the vast amounts of information resources in geography has characteristics of distributed and heterogeneous, how to make use of the characteristics of the grid technology to seek a kind of rule, the organization and scheduling model, the grid resources according to the specific rules of unified organization, scheduling, for the user to provide services, users obtain information resources based and grid technology process needed to solve problems in the resource sharing [3].

Traditional network information resource providers only limited to the local domain and single source, does not have the heterogeneity, organization pattern is relatively simple, number and types of resources available to users are less, there some limitations. At the same time, the traditional streaming media resource scheduling efficiency is low, the response time is long, etc., also seriously affect the resource sharing efficiency [4][5]. Aiming at this problem, this study proposed a local-global teaching resource organization and scheduling model, through to the local server resources constantly monitor. The retrieval results are arranged according to a specific rule, avoid blind search resources, shortened the time to find the resources, effectively improve the resource utilization efficiency and the grid system performance.

\section{Grid theory}

Characteristics of the grid. Grid electricity network is to learn the concept proposed by an emerging information technology. The ultimate goal of the grid is full use of existing resources, to provide users with the sharing of resources and services, including the following features.

1. Distribution

Different sources of information resources in grid environment, and stores around the world, showing distributed. In this distributed grid environment, the need to address organizational issues and storage grid resource scheduling issues and the synergies and interaction issues between the user and system.

2. Heterogeneity

Grid information resource types, quantity, access interface and access rules vary, showing heterogeneity. Vast amounts of information resources may be stored in different types of computer 
systems, with different types of computing interfaces and computing models, therefore, grid technology has the ability to deal with heterogeneous resources.

3. Dynamic

Grid resources are not static, resource provider can always add or remove resources, sharing of resources is constantly changing, dynamic presentation.

Constitution of grid. The application of grid technology to the resource sharing problem, is the essence of the establishment of virtual network environment, integrating the entire network into a super computer can not only realize comprehensive sharing of software, hardware, information, data and other resources, but also can deal with the problem of human-computer interaction, artificial intelligence[6][7]. The structure of information resources based on the grid is shown in Figure 1.

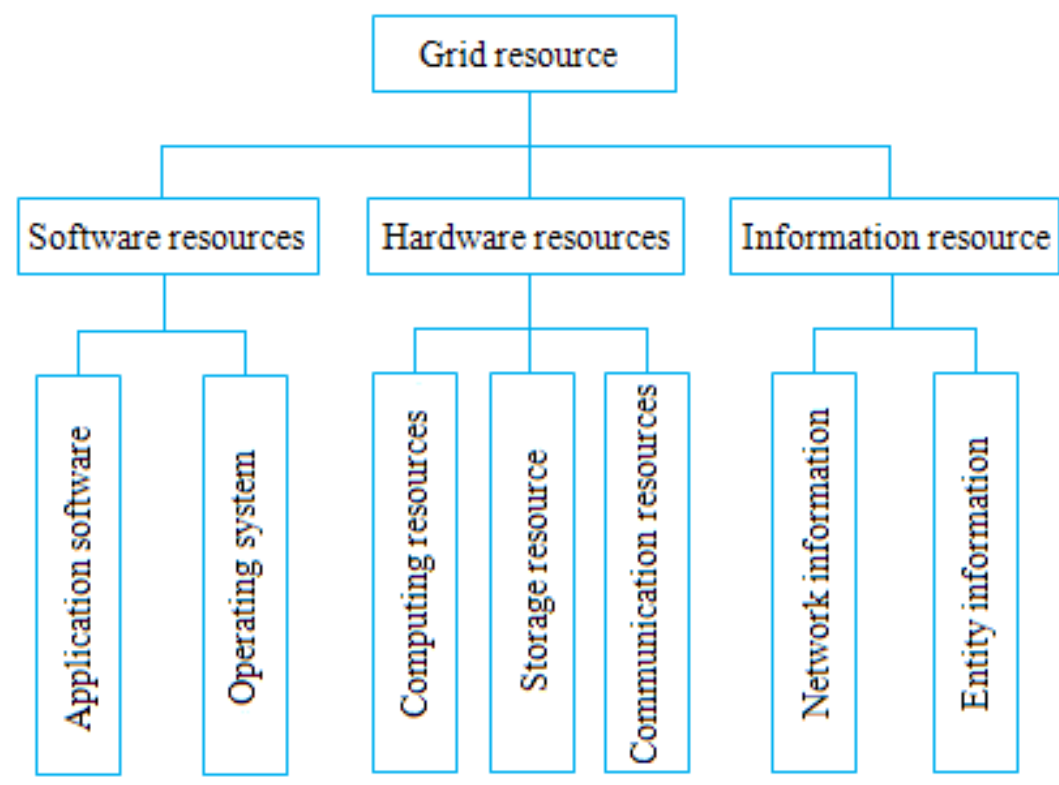

Fig.1 structure of information resources based on grid

\section{Organization mode of teaching resources in the grid environment}

Traditional organization and dispatch model of information resources. Resource organization refers to meet the needs of users of teaching resources, formulate corresponding rules and standards of resource description, naming, arrangement and combination, make it according to the provisions of the form of the distribution and storage, the traditional network information resource provider only limited to the local domain, single source information, organization pattern is relatively simple, unable to realize between the distributed, heterogeneous and dynamic information resources sharing. In this resource organization mode, the number and the types of the resources available for the user are less, and the user has limited the usage of the resources, and the structure is shown in Figure 2. 


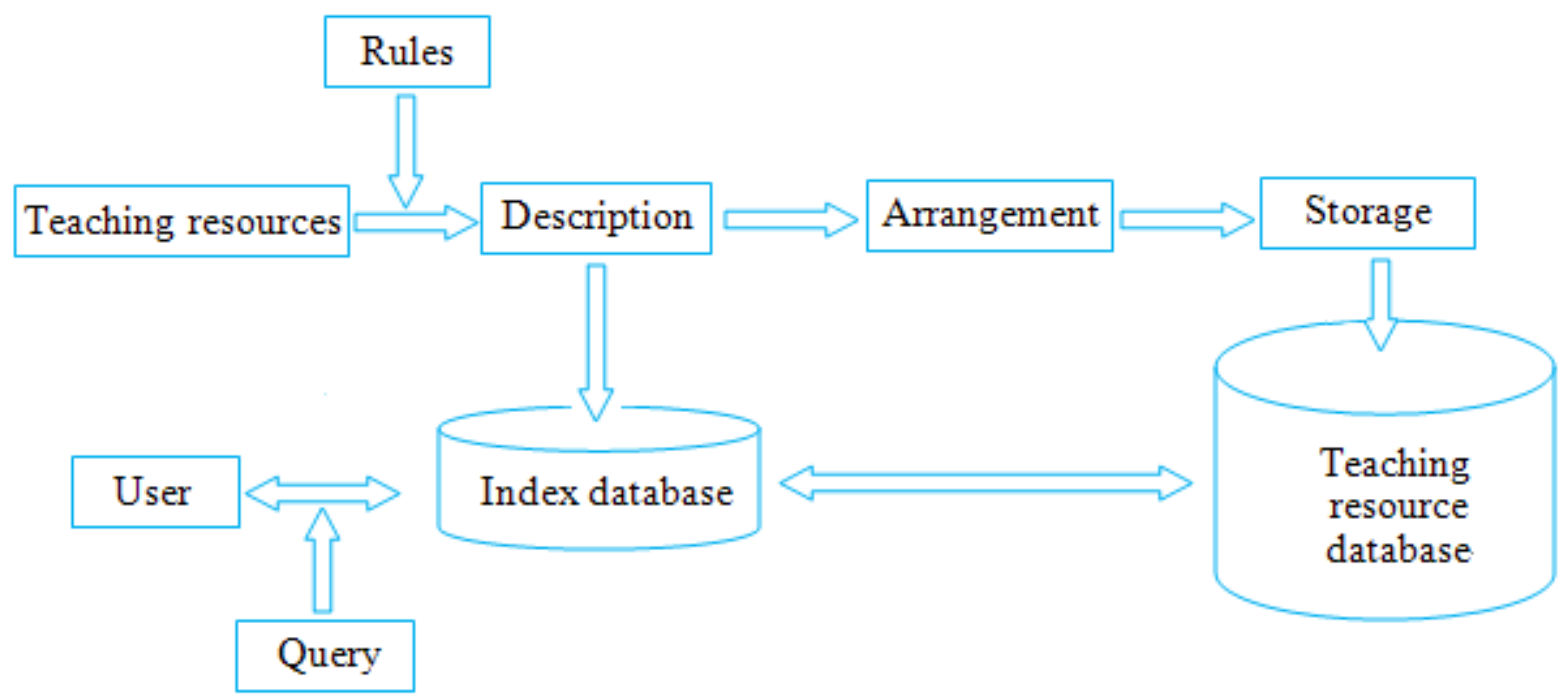

Fig.2 traditional organization and dispatch model of information resources

Resources organization and scheduling model based on grid. Common resources organization and scheduling model with hierarchical structure [8][9], it manage all resources into several function layer, and a layer can use services provided by the next layer of realize its function, strong applicability, its structure is shown in figure 3.

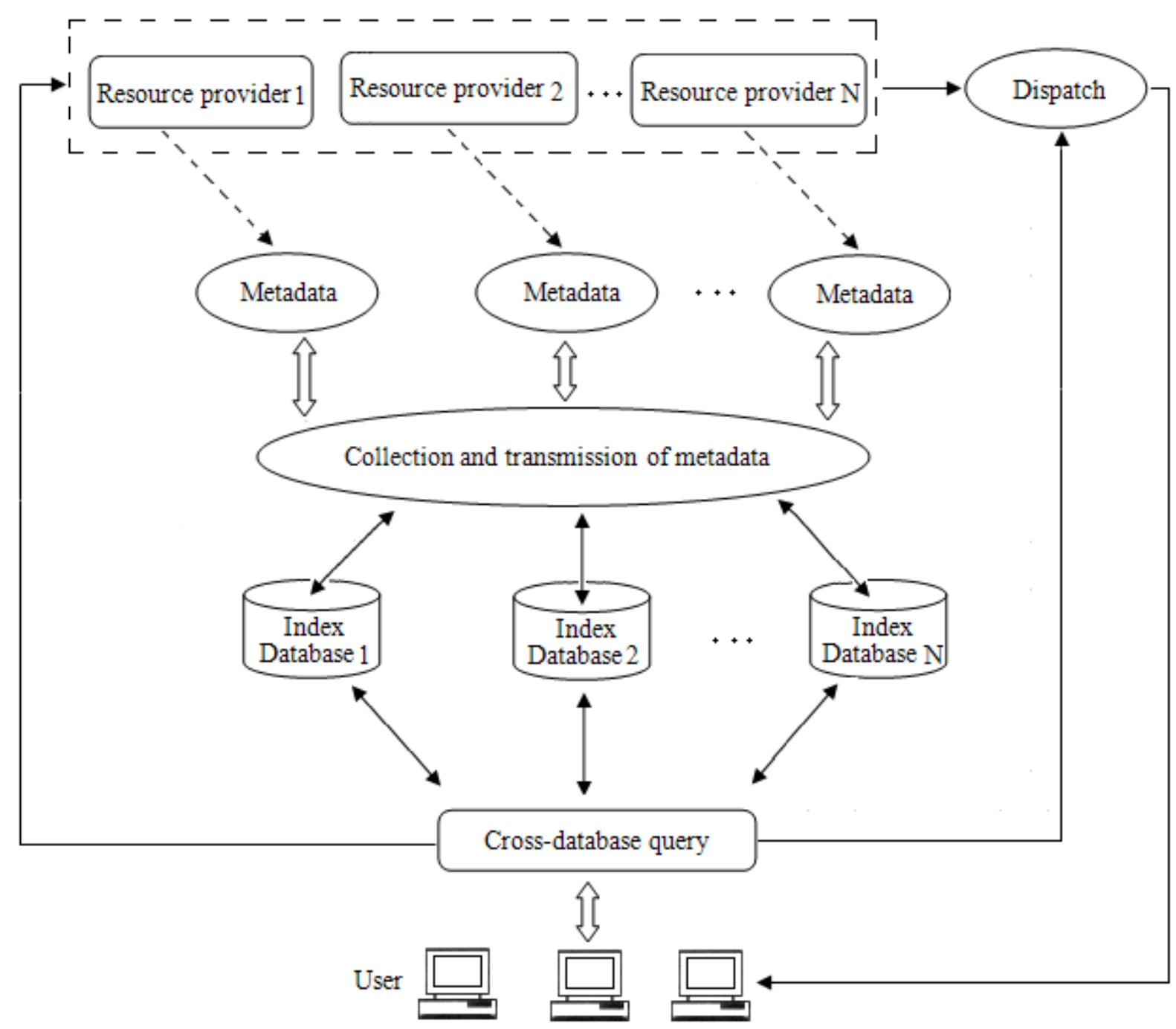

Fig.3 organization and scheduling mode of layered grid resource 
In this resource organization and scheduling mode, users can query every resource in the grid system according to the rules, and share the information resources [10]. However, when a query commands corresponding to multiple resources, the organization and scheduling model will put these information resources are also presented to the user, which will enable a large number of data transmission at the same time, causing blockage of data produce bottleneck effect, serious impact on the query speed, waste the time of the user, it greatly decreases the performance of grid system.

Organization and scheduling model of local--global teaching resource. In view of the problems existing in the grid resource organization and scheduling model, this study design based on a grid of the local-global resource organization and scheduling model. The model consists of three levels of composition, namely grid resource node layer, local resource server layer and the global resource server layer, each local server resources and its corresponding grid resource nodes constitute a separate autonomous resources domain, as shown in Figure 4.

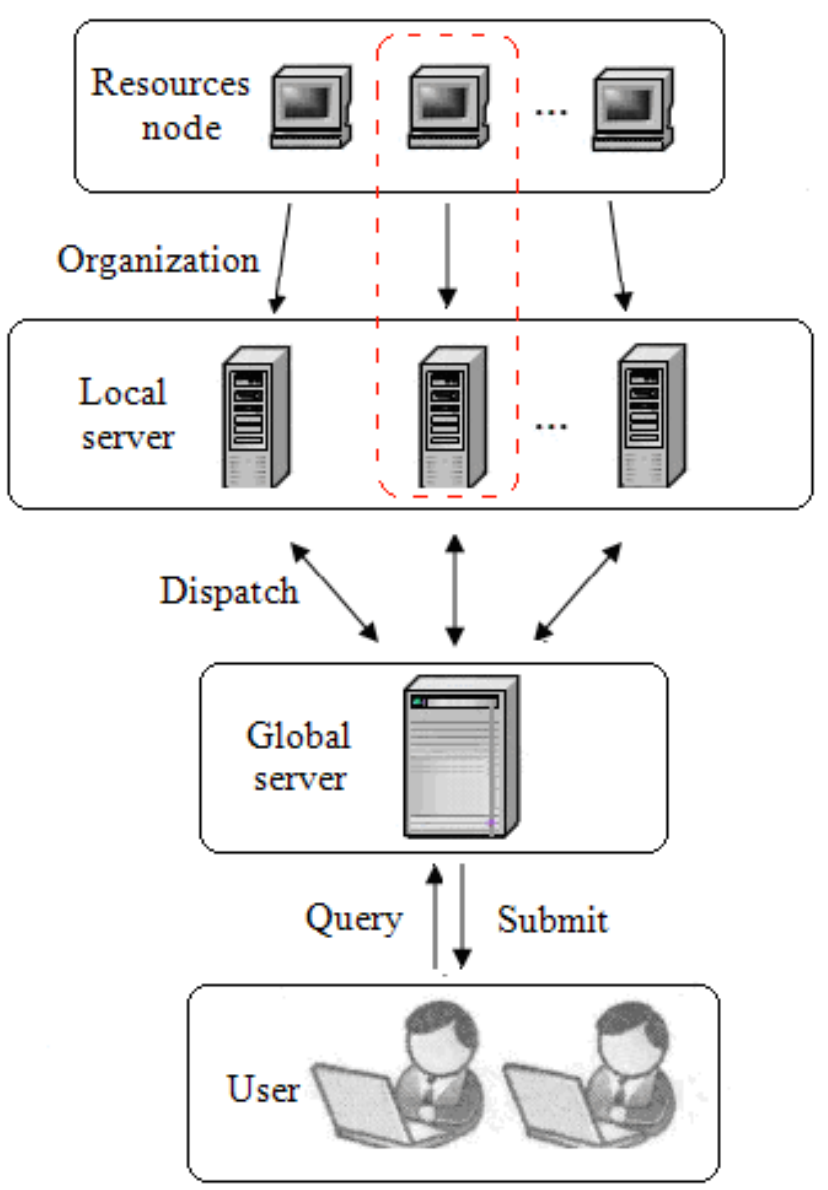

Fig.4 organization and scheduling model of local-global teaching resource based on a grid

The organization mode, according to certain rules of information resources in a resource node is described and named, stored in the corresponding local resources in the server, the server's global resources is responsible for local resources in the server deployment and monitoring, data information has the following advantages:

1. Resource query process adopts parallel query method, improve the query speed when the global resource server receives the user retrieval instructions, according to certain principles are assigned to each autonomous domain to find relevant information, and then to submit the results to the global server to further review, filtering, and scheduling, returned to the user to use the most appropriate results. This approach improves the search speed of teaching resources, save the user's query time.

2. Can realize online monitoring of information resource usage, dynamic submit query results, make the education resource utilization efficiency is greatly increased. Search process may find a lot of the same information, but from different autonomous domain respectively, then the global 
resource server will be according to the monitoring results of each autonomous domain information call situation as well as the local resource server equipment usage analysis and sorting, the call rate, low utilization rate of equipment smallest preferred teaching resources to users.

\section{Conclusion}

Teaching system of teaching resources in grid environment has many characteristics, such as distribution and heterogeneity, reasonable and effective resources organization and scheduling model is the key to improve the efficiency of resource utilization factor. In view of the present teaching resources in the process of scheduling problems, this paper proposes a based on grid technology local-global type teaching resources organization and scheduling model, this model including the global resource server, local server and grid resources nodes three hierarchies, the realization of hierarchical management of resources. When resources query parallel is adopted to avoid the blindness of query information resources, improve the information extracting speed; Global resource server can real-time dynamic monitoring autonomous domain of resource usage, and according to certain rules to submit the retrieval results are arranged, the most suitable for users to use the resources of row in front of the queue, in improving the utilization ratio of resources at the same time reduces the communication bottleneck, reduces the load of server, greatly improving the resources utilization, effectively improve the performance of the grid system.

\section{References}

[1] Chih-Jen $\mathrm{Wu}, \mathrm{Yu}-\mathrm{Wei}$ Chenl. Seamless channel transition for slotted generalized fibonacci broadcasting[J]. Multimedia Systems, 2007, 113(10):2

[2] Alfred W. Loo. The further of Peer-to-Peer Computing An economical method for pumping up computing power tapping into P2P systems using Web server technologies [J]. Communications of the ACM 2003(9):56-61

[3] Liu Fang, Zhao Jing. An Immune Clonal Selection Scheduling Algorithm for Input-Queued Switches[J]. Lecture Notes in Computer Science, 2006(5):42-47

[4] Jiang $\mathrm{Yu}$, Chun Tung Chou. A dynamic caching algorithm based on internal popularity distribution of streaming media [J]. Multimedia Systems, 2006, (12):3

[5] Vitor OK Li, Wajiun Liao. Performance model of interactive video-on-demand Systems[C]. IEEE Jon Selected Are in Comm. 2003,Vol.14(6):1099-1109

[6] Sterck H D, Markel R S, Pohl T, et al. A Lightweight Java Task spaces Framework for Scientific Computing on Computational Grids[C].Proceedings of the ACM Symposium on Applied Computing, Melbourne, Florida, 2003: 1024-1030.

[7] J.Yu, R.Buyya, Grid Computing; Infrastructure, Service and Applications [M].New York: CRC Press, 2009, 119-146.

[8] I. Brandic, S. Benkner, G. Engelbrecht, et al. Quos support for time-critical grid workflow applications. In proceedings of the 1st international conference on e-Science and Grid computing, 2005, 108-115.

[9] R. Sakellariou, H. Zhao, E. Tsiakkouri, et al. Scheduling Workflows with Budget Constraints [J]. Integrated Research in GRID Computing, 2007, 189-202.

[10]S. Abrishami. Cost-Driven Scheduling of Grid Workflows Using Partial Critical Paths [J]. IEEE Transactions on Parallel and Distributed Systems, 2012, 23(8):1400-1414 